\title{
I \\ Sida en Chine : discours \\ et pratiques de la sexualité
}

\author{
La gestion de l'épidémie par l'Etat éclaire et reflète les contradictions de la société
}

EVELYNE MICOLLIER

Les enjeux qui se cristallisent autour de la prévention du virus immunodéficitaire humain (VIH)/

Syndrome immunodéficitaire acquis (Sida) et de la gestion du risque épidémique éclairent des contradictions prégnantes dans la société chinoise. Ils constituent un angle d'approche pertinent pour analyser les transformations en matière de sexualité et appréhender la pluralité des discours et des pratiques. La perspective théorique adoptée dans cet article s'inscrit dans le cadre d'une approche constructiviste ; celle-ci a reformulé le corpus des connaissances relatives à la sexualité en s'interrogeant sur le contexte social et historique de leur production. En Chine, l'histoire épidémiologique des infections sexuellement transmissibles (IST) montre que la gestion de la sexualité importe davantage que le contrôle des maladies ou l'éducation pour la santé.

$\mathrm{L}$ es enjeux qui se cristallisent autour de la prévention du VIH/Sida et de la gestion du risque épidémique éclairent des contradictions prégnantes dans la société chinoise : ils constituent un angle d'approche pertinent pour analyser les transformations en matière de sexualité et appréhender la pluralité des discours et des pratiques qu'elles induisent ${ }^{(2)}$. Comme l'a expliqué Alain Giami s'interrogeant sur les déterminants et les contextes des conduites sexuelles ${ }^{(3)}$, « le phénomène du Sida fait apparaître des problématiques nouvelles dans le champ de la sexualité : les réseaux de partenaires sexuels et la notion de risque, qui constituent la marque d'une évolution des constructions sociales de la sexualité ». Ainsi, dans le contexte de l'épidémie de Sida, une reconstruction heuristique du « comportement sexuel » se produit : dans les études, certains comportements sont retenus au détriment d'autres en fonction de leur impact sur la transmission du VIH, et sur un ensemble de pratiques considérées comme non-sexuelles, certaines
Cependant, en dépit de la volonté politique des acteurs officiels, de la prégnance de valeurs familiales et de traits culturels hérités du passé éventuellement «revitalisés », les personnes sont soumises à des influences multiples associées au processus de globalisation et à l'adhésion à un modèle de société consumériste. Des études récentes indiquent des changements notables en matière de sexualité. L'article est organisé en trois parties : la première est une revue analytique de la littérature scientifique, et des données relatives aux comportements sexuels et aux représentations de la sexualité. La deuxième porte sur l'action et le discours du gouvernement, approchés sous l'angle de la dynamique de l'épidémie de Sida et du risque de transmission sexuelle. Enfin, dans la troisième partie, la gestion de la sexualité par l'Etat est analysée dans une perspective diachronique ${ }^{(1)}$.

sont sélectionnées selon ce même critère. La perspective théorique adoptée dans cet article s'inscrit dans le cadre d'une approche constructiviste qui a reformulé le corpus de connaissances relatives à la sexualité en s'interrogeant sur le contexte social et historique de la production de ce

1. Cet article est la version révisée d'une communication présentée au colloque organisé par le Centre d'études sur la population et le développement (CEPED) Laboratoire population-environnement-développement (LPED), « La santé de la reproduction confrontée au Sida : enjeux sociaux et comportementaux dans les pays du sud », IRD-ANRS, Nogent-sur-Marne, France, 22-23 novembre 2004.

2. Cet article s'appuie sur des enquêtes portant sur le commerce du sexe et le risque de VIH/Sida menées en 1997 et en 1999 en Chine populaire (Shanghai, Guangxi : ville de Beihai) et à Taiwan (Taipei) dans une perspective anthropologique (entretiens, observations sur les sites où se pratiquent régulièrement des échanges sexuels marchands, récits de vie construits avec des travailleuses du sexe), cf. Evelyne Micollier (éd.), Sexual Cultures in East Asia. The Social Construction of Sexuality and Sexual Risk in a Time of AIDS, Londres, RoutledgeCurzon, 2004.

3. Alain Giami, « De Kinsey au Sida : l'évolution de la construction du comportement sexuel dans les enquêtes quantitatives ", Sciences sociales et santé, vol. $9, \mathrm{n}^{\circ} 4$, 1991, pp. 23-56. 

jour. L'évolution des stratégies officielles de lutte contre le Sida montre l'institutionnalisation croissante de la prévention et la réappropriation par l'Etat d'interventions sanitaires initiées par un réseau associatif dépendant largement des organismes onusiens et des ONG internationales. Cette réappropriation politique de la lutte contre le Sida s'accompagne d'un discours visant à contrôler les pratiques et les représentations liées à la sexualité.

Mais, en dépit de cette volonté politique et de la prégnance de valeurs familiales et de traits culturels hérités du passé, les individus sont soumis à des influences multiples associées au processus de globalisation et à l'adhésion à un modèle de société consumériste. Des changements notables en matière de sexualité sont confirmés par les études récentes sur la sexualité ${ }^{(9)}$ et par les données du rapport national ${ }^{(10)}$. A propos de ce dernier, Erwin J. Haeberle, sexologue allemand qui a contribué à sa conception au niveau théorique et méthodologique, explique : «En général, les résultats des grandes enquêtes quantitatives sur les comportements sexuels sont presque prévisibles dans la mesure où ils sont similaires d'un pays à un autre. Par exemple, le fait qu'une grande majorité d'étudiants (premier cycle d'études universitaires) perçoive l'éducation sexuelle comme trop conservatrice révèle des sentiments identiques à un niveau global dans notre monde en mutation rapide. Le fait qu'un nombre considérable de personnes ait eu des rapports pré-maritaux est également un résultat quasi universel » ${ }^{(11)}$.

Haeberle souligne aussi que le rapport Liu, première grande étude quantitative sur les comportements sexuels, génère plus de questions que de réponses ; il ouvre ainsi la voie à la recherche sur la sexualité en Chine.

Les modèles idéologiques et comportementaux qui sous-tendent la vie sexuelle des individus sont transmis par des messages émanant de la famille, de l'Etat, relayés par l'école ou la planification familiale ; le triomphe du consumérisme et la globalisation économique et culturelle sont à l'origine de nouveaux messages. Trois systèmes de représentations et de pratiques coexistent donc au sein de la société chinoise contemporaine et génèrent des tensions au niveau des valeurs, des comportements et des actions. Le premier système est issu de la tradition chinoise plurielle et en constante évolution ${ }^{(12)}$; le second a été introduit par l'idéologie marxiste et a nourri le projet de construction d'une société socialiste; le dernier peut être associé à un modèle global qui concerne la Chine comme le reste du monde.

Notre article est organisé en trois parties. La première partie est une revue analytique de la littérature et des données dont nous disposons relatives aux comportements sexuels et aux représentations de la sexualité. La deuxième envisage l'action et le discours des autorités sous l'angle de la dynamique de l'épidémie de Sida et du risque de transmission sexuelle. Enfin, la préoccupation de gestion de la sexualité par l'Etat est analysée dans une perspective diachronique dans la troisième partie. Notons d'emblée que l'existence même des travaux qui seront évoqués dans la première partie est un signe de changement. Un certain nombre d'entre eux émanent d'une demande officielle d'expertise et d'informations au service de l'action gouvernementale dans les domaines de la santé, de l'éducation sexuelle et de la planification familiale ; bien que controversés, ces travaux ont obtenu le soutien d'organisations officielles.

\section{Sexualité dans une Chine en transition}

\section{Rapports sur la sexualité et autres études : vers une reconnaissance officielle des problèmes}

En matière d'études sur la sexualité, on distingue les grandes études quantitatives qui suivent plus ou moins le modèle du rapport Kinsey ${ }^{(13)}$ et des études plus fines qui utilisent des données qualitatives comme matériel principal. Le rapport

9. Voir Pan Suiming, «A Sex Revolution in current China », Journal of Psychology and Human Sexuality, n 6, 1993, pp. 1-14 ; James Farrer, Opening Up: Youth Sex Culture and Market Reform in Shanghai, Chicago, University of Chicago Press, 2002; Gail Hershatter, « Sexing Modern China » in Gail Hershatter, Emily Honig, Jonathan N. Kipman and Randall Stross (éd.), Remapping China. Fissures in Historical Terrain, Stanford, Stanford University Press, 1996, pp. 77-93; Xu Xiaoqun, «The Discourse on Love, Marriage, and Sexuality in Post-Mao China: A Reading of the Journalistic Literature on Women », Positions. East Asia Cultures Critique vol. 4, n 2, 1996, pp. 381-414.

10. Liu Dalin, Ng Man-lun, Zhou Liping et Erwin J. Haeberle, Zhongguo dangdai xing wen hua : Zhongguo liangwan lie 'xing wenming' diaocha baogao (Contemporary Chinese Sexual Culture: Report of the 'Sex Civilization' Survey on 20000 subjects), Shanghai, Sanlian shudian, 1992

11. Préface de Erwin J. Haeberle dans Liu Dalin, Ng Man-lun, Zhou Liping and Erwin J. Haeberle, Sexual Behavior in Modern China. Report on the Nationwide Survey of 20000 Men and Women, New York, Continuum, 1997, p. 11.

12. J'adopte ici une approche dynamique de la tradition englobant une analyse de ses permanences et de ses ruptures au cours de l'histoire, de son 'invention' (Eric Hobsbawm et Terence Ranger (éds), The Invention of Tradition, Cambridge, Cambridge University Press, 1983) et de sa revitalisation actuelle qui peut s'expliquer à deux niveaux — par l'émergence au niveau social d'une condition post-moderne (Jean-François Lyotard, $\mathrm{La}$ Condition post-moderne, Paris, éd. de Minuit, 1979) et au niveau psychologique d'une 'conscience contradictoire' (contradictory consciousness : Matthew C. Gutmann, The Meanings of Macho: Being a Man in Mexico City, Berkeley, University of California Press, 1996, p. 15, s'inspirant du concept de Gramsci) d'un certain nombre de membres de la société.

13. Alfred Kinsey et al., Le comportement sexuel de l'homme, Paris, éditions du Pavois, 1948 ; Kinsey et al. Le comportement sexuel de la femme, Paris, Amiot-Dumont, 1953, (traductions françaises). 
national sur la sexualité paru en 1992, couramment évoqué comme le rapport Kinsey chinois (rapport Liu), et ses analyses critiques donnent un éclairage significatif de la pluralité des discours sur la sexualité. Au moment où, en 1989-1990, Liu Dalin coordonne des études quantitatives, le rapport Kinsey américain est traduit en chinois par le sociologue Pan Suiming qui dirige l'Institut de recherches sur le genre et la sexualité au sein du Département de sociologie de l'Université du peuple de Pékin. Pan aurait communiqué sa traduction à Liu qui devait prendre le rapport Kinsey comme modèle méthodologique ${ }^{(14)}$.

A la différence de rapports récents sur la sexualité dans d'autres pays, le rapport Liu n'a pas de relation directe avec la transmission sexuelle du VIH puisque, au début des années 1990, quand l'enquête est menée, le risque épidémique de Sida était nié. Cependant, les IST, dont le Sida, avaient été identifiées comme un problème lié à la sexualité et devant être traité dans le cadre d'interventions sanitaires et sociales.

Le rapport Liu s'appuie sur une enquête conduite auprès de 20000 personnes dans quinze provinces ${ }^{(15)}$, organisée par le Centre de recherches sociologiques de sexologie de Shanghai fondé en décembre $1988^{\left({ }^{(1)}\right.}$. L'enquête cible quatre catégories de population : les jeunes (collégiens et lycéens ; étudiants à l'université), les couples mariés et les auteurs de crimes sexuels ${ }^{(17)}$. Lobjectif est d'identifier les problèmes liés à la sexualité des personnes, en mettant l'accent sur les adolescents et les jeunes, le contrôle et la gestion de la sexualité, et la propagation des IST. L'enquête est réalisée grâce au soutien de nombreux acteurs privés et publics : des entreprises chinoises et multinationales, des organisations de masse telles que la Planification familiale, la Fédération des femmes, le Syndicat des travailleurs, et des institutions étatiques comme les départements de police ou les écoles ${ }^{(18)}$, par l'intermédiaire desquels les questionnaires sont distribués.

Le climat politique et social à la fin des années 1980 était favorable à l'élaboration et au lancement d'une telle enquête. Des médecins, en particulier le docteur $\mathrm{Wu}$, directeur de l'Académie des sciences médicales, et le professeur Ruan de l'Université médicale ${ }^{(19)}$, tous deux à Pékin, étaient alors des précurseurs de la recherche sexologique. En outre, après une décennie de réforme et d'ouverture, les problèmes associés à la sexualité et les lacunes en matière d'éducation sexuelle sont reconnus officiellement et bénéficient d'une certaine visibilité.

Nous retiendrons les dix principaux résultats de cette enquête nationale qui concernent notre objet de recherche, en reprenant les termes utilisés par les auteurs ${ }^{(20)}$.
1. L'augmentation du nombre de relations amoureuses parmi les jeunes collégiens et lycéens. Selon une enquête annuelle menée à Shanghai entre 1985-88, environ $60 \%$ des lycéens et un pourcentage significatif de collégiens vivaient des histoires d'amour.

2. L'accroissement des rapports sexuels pré-maritaux. Selon les statistiques de plusieurs hôpitaux, il y a eu en 1986 un accroissement de $16 \%$ des grossesses prémaritales et des demandes d'avortement en un an.

3. L'accroissement des rapports sexuels extra-maritaux. Selon plusieurs enquêtes, à partir de la fin des années 1980, le nombre de divorces pour adultère représente entre 25 et $40 \%$ de l'ensemble des divorces ${ }^{(21)}$.

4. L'augmentation des cas déclarés de dysharmonie sexuelle parmi les couples mariés. En 1984, à Shanghai, elle était la cause de $24 \%$ des divorces. En 1986, ce taux a presque doublé $(45 \%)^{(22)}$.

5. L'accroissement des crimes sexuels. Selon le bureau des statistiques de Shanghai, entre 1979 et 1983, le nombre de viols déclaré est multiplié par 4 et l'âge des violeurs diminue. Les viols collectifs sont plus nombreux, plus violents et prémédités ${ }^{(23)}$.

6. L'augmentation du commerce sexuel. Depuis la réforme, le commerce du sexe s'est étendu des villes

14. Communication personnelle de Pan Suiming, 2005. Rappelons qu'en Chine, deux instituts de recherches sociologiques se sont spécialisés dans les études sur la sexualité depuis une vingtaine d'années, celui de Liu Dalin à Shanghai et celui de Pan Suiming à Pékin.

15. Heilongjiang, Liaoning, Pékin, Tianjin, Shandong, Shanxi, Jiangsu, Shanghai, Zhejiang, Fujian, Guangdong, Henan, Sichuan, Xinjiang et Ningxia.

16. Aujourd'hui, ce centre n'est plus dirigé par Liu Dalin professeur retraité qui a créé un musée sur la culture sexuelle (性文化博物馆, xing wenhua bowuguan). Implanté rue de Nankin à Shanghai, l'une des plus commerçantes de la ville, il a dû fermer ses portes en 2001, trois ans après son ouverture, en raison de difficultés financières, le prix de location des locaux étant devenus prohibitifs. De plus, les panneaux publicitaires sur la rue utilisant le terme « sex» (xing) avaient été interdits. Il a depuis réouvert à Yongli (Jiangsu). Voir Liu Dalin, Fushi yu chunmeng: Zhongguo yu Riben de xing wenhua bijiao (Ere de la légèreté et rêve de printemps : comparaison des cultures sexuelles chinoise et japonaise), Pékin, Zhongguo youyi chuban gongsi, 2005.

17. Cette catégorie incluait les violeurs, et des acteurs de l'industrie du sexe (les travailleuses du sexe et les proxénètes).

18. Liu évoque des difficultés imprévues, notamment financières, pour mener à bien le projet survenues après les événements de 1989 à cause des restrictions en matière d'aide financière étrangère imposées à la Chine et de la récession économique (Liu et al., 1997, p. 24). De plus, des campagnes nationales contre le capitalisme et l'obscénité n'ont pas facilité la tâche des enquêteurs, mais en dépit de tous ces obstacles, le projet a été poursuivi.

19. Ruan Fangfu, Sex in China. Studies in Sexology in Chinese Culture, New York, Plenum Press, 1991

20. Liu et al., op.cit., pp. 20-21

21. Liu Dalin, Xing shehui xue (Sex Sociology), Jinan, Shandong renmin chubanshe, 1986.

22. Liu, op.cit.

23. Liu, ibid. 
côtières vers les villes moyennes de la Chine centrale et vers les villages. En 1987, le nombre de prostituées arrêtées a été multiplié par 240 par rapport à 1979.

7. La propagation des IST dont le Sida. A la fin des années 1980, le taux de IST augmente chaque année de $300 \%$ et le Sida se propage alors que le premier cas d'infection a été recensé en 1985.

8. L'augmentation des publications pornographiques. Des campagnes officielles sont périodiquement lancées pour éradiquer la pornographie, confirmant la diffusion de ces matériaux.

9. La confusion des représentations de la sexualité. En Chine, des « idées féodales » sur la sexualité ont encore une certaine influence. De plus, ont été diffusées pendant un siècle les idées de l'Occident capitaliste, auxquelles se sont agrégées des enseignements communistes de la nouvelle Chine, transformées une nouvelle fois par les dernières modes et manières en provenance de l'Europe et des Etats-Unis : un tel éclectisme idéologique aboutit de manière prévisible à une grande confusion pour les jeunes générations. La Chine recherche toujours une manière nouvelle, saine et rationnelle de voir la sexualité ${ }^{(24)}$.

10. La planification familiale connait de grandes difficultés : l'objectif est de maintenir la population en deçà de la barre de 1,2 milliard, mais étant donné la difficulté à éduquer les personnes et à appliquer la politique de planification familiale dans les régions reculées, la probabilité d'un échec est grande. Une raison de plus pour le gouvernement et la population de reconnaitre l'importance de l'éducation sexuelle.

Le discours officiel souligne « la nécessité historique de confronter et de résoudre ces problèmes bien qu'ils soient difficiles à admettre, en particulier le développement du commerce sexuel et la propagation des IST » ${ }^{(25)}$. L'un des arguments mobilisé pour inciter à l'action gouvernementale fait le lien avec la modernisation de la société, suggérant que l'état des relations sexuelles est un indicateur du degré de modernité ${ }^{26)}$.

Selon Judith Farquhar ${ }^{27)}$, le rapport Liu s'inscrit dans une démarche de recherche finalisée aux résultats pré-programmés qui révèle son instrumentalisation au service d'une "pédagogie nationale ». Elle remarque les liens étroits entre les résultats et le programme social préalable qui sous-tend l'intervention gouvernementale en matière d'éducation à la santé et à la sexualité. Le titre, qui utilise les termes de wen hua (文化, culture) et wenming (文明, civilisation), situe le projet dans le cadre de l'idéologie du développement national ${ }^{(28)}$. La brève préface au questionnaire distribué aux participants est significative : "La sexualité est une composante majeure de la vie humaine intimement liée à un développement sain de la jeunesse, au bonheur et à l'harmonie des couples mariés, enfin à la stabilité et à la cohésion sociales. Aujourd'hui, la sexualité pose de nombreux problèmes en premier lieu parce que la société avait produit des inhibitions sexuelles et également pour d'autres raisons. Cette recherche est mise en œuvre pour comprendre la situation et pour trouver des moyens de résoudre les contradictions. Elle aura une grande portée sur le développement social ; nous sollicitons donc votre aide et votre soutien $»^{(29)}$.

William R. Jankowiak est le premier à avoir conduit une recherche ethnographique sur les comportements sexuels et amoureux en Chine (à Hohhot, capitale de la Mongolie intérieure). Il explique en particulier comment des changements dans le cadre de la vie quotidienne, notamment les relations sexuelles et amoureuses, transforment les relations sociales traditionnelles. Son appréciation du rapport Liu est très positive car, selon lui, ses auteurs ont tenté une comparaison avec les autres enquêtes similaires (conduites au Japon, aux Etats-Unis, en Europe de l'Ouest) ; de plus, leurs données peuvent être exploitées par d'autres chercheurs parce qu'ils se sont intéressés aux comportements sexuels des hommes et des femmes en prenant soin de définir la sexualité comme un construit social ${ }^{(30)}$. Jankowiak souligne l'intérêt d'approcher les personnes à la fois en tant qu'individus et en tant que sujets relationnels, une perspective qu'il adopte dans ses propres travaux.

Ainsi, au cours des années 1980 et 1990, des enquêtes élaborées suivant la méthodologie du rapport Kinsey sont menées aussi bien en Chine que dans les autres sociétés de culture chinoise soumises à des transformations socioéconomiques et culturelles rapides (à Taiwan et à Hong

24. Fan Minsheng, "Analysis of the Cause for the Resurgence of Sexually Transmitted Diseases », Shehui xue (Sociology), n 12, 1990, p. 27.

25. Liu et al., 1997, p. 21.

26. Gail Hershatter, op. cit., p. 90 .

27. Judith Farquhar Appetites. Food and sex in post-socialist China, Durham, Duke University Press, 2002, pp. 226-227.

28. Pour une discussion sur la relation entre ces termes, voir Anna Anagnost, National PastTimes: Narrative, Representation, and Power in Modern China, Durham, Duke University Press, 1997, p. 79, citée par Farquhar, op.cit., p. 227 : la culture nationale doit être reformulée pour servir un projet de civilisation qui prend pour modèle les sociétés industrielles avancées occidentales et asiatiques.

29. Liu et al., op.cit., 1992, p. 774.

30. William R. Jankowiak, Sex, Death, and Hierarchy in a Chinese City. An Anthropological Account, New York, Columbia University Press, 1993, p. 35. 


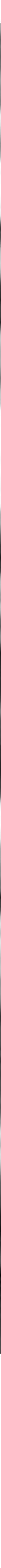

Kong). Chang Jui-shan ${ }^{(31)}$, sociologue spécialisée dans des travaux comparatifs sur la sexualité et les rapports sociaux de sexe (genre), déplore que la problématique se résume souvent à identifier si les sociétés suivent ou non le chemin emprunté par l'Occident en matière de sexualité.

Chang Jui-shan porte un regard critique en identifiant les lacunes de ces « rapports Kinsey chinois » ${ }^{(22)}$. Selon elle, ces derniers ne sont d'aucune utilité pour expliquer les faits suivants tirés de ses propres recherches :

- Pourquoi les collégiens de Hong Kong décrivent des comportements sexuels plus permissifs, tout en ayant des idées plus conservatrices que les collégiens taiwanais?

- Pourquoi les étudiants à l'université en Chine sont-ils plus permissifs que ceux de Hong Kong en matière d'expérimentation sexuelle, alors que Hong Kong est plus moderne et occidentalisé ?

- Pourquoi la Chine connaitrait-elle une " révolution sexuelle » alors que Taiwan et Hong Kong vivraient des changements moins drastiques en matière de mœurs sexuelles?
- Pourquoi les jeunes Taiwanais revendiquent-ils leur conservatisme sexuel, affirmant qu'ils ne veulent pas devenir « dépravés » comme les Américains, tandis que les jeunes de Chine populaire conçoivent une sexualité plus débridée comme un aspect de leur modernité ?

Pour tenter de répondre à ces questions, Chang propose un paradigme qui distingue deux niveaux d'analyse: l'un s'appuie sur la nature même de la sexualité avec des normes définies par une tradition culturelle ; l'autre porte sur les pratiques sexuelles qui émergent en réponse aux différentes « infra-structures », selon la période historique et la société au sein d'une même culture. Le premier niveau permet des comparaisons inter-culturelles et le deuxième des compa-

31. Chang Jui-shan et al., « Pre-marital sexual mores in Taiwan and Hong Kong: two pathways to permissiveness », Journal of Asian and African Studies, vol. 32, n ${ }^{3}-4,1997$, pp. 265-287 ; Chang Jui-shan, « Scripting Extramarital Affairs: Marital Mores, Gender Politics, and Infidelity in Taiwan », Modern China, vol. 25, n 1, 1999, pp. 69-99.

32. Chang Jui-shan, « Do we need 'Kinsey Reports' in Chinese Societies? An Alternative Paradigm for the Study of Chinese Sexuality », Bulletin of Concerned Asian Scholars, vol. 31, n 1,1999 , pp. 40-42. 
raisons intra-culturelles. Des hypothèses de travail que ne prennent pas en compte les rapports de style Kinsey sont envisagées : à Hong Kong, l'impact du christianisme et du passé colonial britannique ; à Taiwan, une idéologie confucéenne revitalisée et instrumentalisée par l'Etat visant à promouvoir une certaine construction de l'identité nationale ; en Chine, un certain degré d'anomie et de doute au niveau des valeurs, induits par la diversité des idéologies et des modèles de comportements - dans une société où certaines catégories de la population expérimentent une condition post-moderne ${ }^{(33)}$.

Dans les rapports de type Kinsey, les auteurs ne se préoccupent pas d'identifier les constructions locales de la sexualité ; leur question implicite de départ consiste à appréhender le degré de permissivité ou de répression sexuelle des cultures ou des individus, analysé à partir de données statistiques portant sur une sélection de pratiques sexuelles. Cette question par sa référence implicite à «l'hypothèse répressive », un concept freudien critiqué par Michel Foucault, révèle l'inscription dans une conceptualisation occidentale et moderne de la sexualité ${ }^{(34)}$. Dans l'Occident moderne, l'influence freudienne a placé le sexe au centre de la vie et la sexualité est « socialisée » dans le sens de l'affirmation et de la présentation de soi.

Notre démarche -citant les travaux de Chang et exposant son questionnement - se décentre d'une perspective positiviste et considère ces travaux comme des méta-discours sur la sexualité. Que ses hypothèses soient confirmées ou non, et même si la méthodologie est l'objet de controverses, elles recèlent un questionnement des études chinoises sur la sexualité dans une perspective comparative ; ces productions témoignent éventuellement d'un processus « d'indigénisation » d'études « objectives » formatées pour être conduites en Occident, Chang étant elle-même une « intellectuelle en diaspora ${ }^{(35)}$.

Selon nous, la critique faite par Chang de l'enquête Liu modelée sur le rapport Kinsey, et en particulier de l'utilisation de l'hypothèse répressive de Foulcault et des concepts freudiens de la sexualité dans l'élaboration du cadre théorique de l'enquête, est tout à fait fondée. Dans le contexte d'une étude menée auprès d'informateurs chinois, la pertinence de tels concepts et leur traduction méthodologique par exemple sur la trame du questionnaire ou sur les consignes données aux participants pour répondre, méritent d'être discutées.

Une discussion du même ordre s'est engagée entre chercheurs travaillant sur la question de l'identité sexuelle : la pertinence d'une identité "gay/lesbienne/queer» aujourd'hui «mondialisée » mais produit de mobilisations collectives récentes dans des sociétés occidentales, se pose dans les sociétés chinoises où les spécialistes préfèrent parler de « personnes qui ont des pratiques homosexuelles » en se focalisant pour la catégorisation d'un point de vue distinctif sur les pratiques plutôt que sur l'identité ${ }^{(36)}$.

Enfin, évoquons brièvement l'étude pionnière de Farrer sur la sexualité de la jeunesse à Shanghai. Pour lui, les changements en matière de sexualité sont à relier plus directement à la réforme, à la transition vers une économie de marché qui a permis le développement du consumérisme : en mettant en évidence les relations dialectiques de la culture sexuelle avec l'émergence d'institutions et de pratiques spécifiques à une économie de marché, il privilégie le marché comme facteur explicatif de la culture sexuelle shanghaienne ${ }^{(37)}$. L'étude ethnologique de Farrer porte sur la culture sexuelle (性文化, xing wenhua) des jeunes hétérosexuels de Shanghai âgés de 18 à 35 ans, leurs rencontres, leurs histoires sexuelles et les discours publics sur la sexualité. Farrer analyse comment ces pratiques sociales se sont transformées pendant la période des réformes de 1980 à 2000. Le récit le plus populaire d'une culture sexuelle en transition est l'histoire d'une « ouverture » vers un modèle de culture sexuelle plus libérale sous l'influence de l'Occident et du Japon au moyen des loisirs, des médias, et des échanges personnels ${ }^{\left({ }^{38)}\right.}$. Cette histoire est rapportée d'une manière plus nuancée dans les travaux universitaires qui documentent le processus de globalisation sexuelle ${ }^{(39)}$; à partir d'observations ethnographiques dans les discothèques,

33. Je fais référence ici à la condition décrite par Lyotard, op. cit.

34. Michel Foucault, Histoire de la sexualité I. La volonté de savoir, Paris, Gallimard, 1976, pp. 25-67.

35. D'origine taiwanaise, Chang a obtenu son doctorat à l'Université du Michigan et est actuellement en poste à l'Université de Melbourne. Sur cette notion et le mouvement d'indigénisation des savoirs en anthropologie face à la globalisation, cf. Jacky Assayag et Véronique Bénéï (éds.), « Intellectuels en diaspora et théories nomades », L’Homme, $\mathrm{n}^{\circ}$ 156, 2000 ; Gregory Guldin et Zhou Daming (éds.), « Moving from Indigenization towards Globalization », Chinese Sociology and Anthropology, vol. 33, n 1, 2000.

36. Voir, par exemple: Zhou Huashan (Chou Wah-shan), « Homosexuality and the Cultural Politics of Tongzhi in Chinese Societies", in Gerard Sullivan and Peter A. Jackson (éds.), Gay and Lesbian Asia: Culture, Identity, Community, New York, Haworth, 2001 ; Zhou Huashan, Tongzhi: Politics of Same-Sex Eroticism in Chinese Societies, New York, Haworth, 2000 ; Lisa Rofel « Qualities of Desire: Imagining Gay Identities in China », Gay Lesbian Quarterly, vol. 5, $\mathrm{n}^{\circ}$ 4, 1999, pp. 451-74 ; Sang Tze-lan D., The Emerging Lesbian. Female Same-Sex Desire in Modern China, Chicago, University of Chicago Press, 2003.

37. James Farrer, op. cit., p. 10.

38. Farrer, op. cit., p. 11.

39. Dennis Altman, Global Sex, Chicago, University of Chicago Press, 2001 ; James Farrer, « Disco 'Super-Culture': Consuming Foreign Sex in the Chinese Disco », Sexualities, vol. $2, \mathrm{n}^{\circ} 2$, mai 1999 , pp. 147-166. 
Farrer met en évidence que le phénomène social de la « disco»(迪斯可, disike) relève moins d'un processus de « localisation » ou de subversion de pratiques globales que d'une consommation active et d'une participation à une sorte de « cosmopolitisme » sexuel, et qu'il s'inscrit davantage dans la construction d'une « super-culture » que d'une « sous-culture ».

Pour conclure cette revue limitée mais à visée heuristique de la littérature sociologique et ethnologique récente sur la sexualité, soulignons que la sexologie est devenue un domaine de recherche discret dans la Chine des réformes. Selon le sociologue Pan Suiming, plus de deux cents livres de sexologie étaient en circulation en 1991 contre seulement six en 1979. Le discours scientifique de la sexologie a produit un langage neutre pour l'expression de la sexualité et de l'éducation sexuelle et a donc facilité la communication dans ce domaine ${ }^{(40)}$. Il met en relation la diversification, la visibilité et éventuellement l'augmentation de l'activité sexuelle avec de nombreux facteurs ; significatif est notamment l'impact de la transformation de l'habitat - les changements qui se sont produits en matière de logement, à savoir l'abandon des maisons sur cour au profit d'appartements dans des immeubles où la vie privée est spatialement mieux séparée de la vie collective. Ainsi, le mode de vie des familles et des individus s'est privatisé permettant plus d'intimité et une expression plus ouverte de la sexualité ; alors que dans la société traditionnelle, le fondement de la moralité sexuelle était lié au contrôle stable et inséparable qu'exerçait le groupe sur l'individu ${ }^{(41)}$.

\section{Discours néo-traditionnels : représentations de la sexualité}

Le corpus ancien de connaissances sur la sexualité comprend des arts érotiques légitimés par des discours médicaux ou religieux et qui sont qualifiés de manière vague comme d'obédience taoïste ou confucianiste. A ce sujet, mentionnons l'apport de l'ouvrage pionnierde référence de Robert van Gulik ${ }^{(42)}$ qui proposa une synthèse des origines jusqu'à la fin de la dynastie des Ming (1644). Selon Charlotte Furth ${ }^{(43)}$, ce corpus fut une première fois revitalisé au début du XX $X^{\mathrm{e}}$ siècle avec la ré-introduction de la sexualité dans le discours public au service d'un projet de modernisation de la société où la santé de la nation est conçue comme liée à la capacité de reproduction biologique de la population. Il est aujourd'hui une nouvelle fois remobilisé pour démontrer que les représentations de la sexualité en Chine se distinguent de celles qui prévalent dans le monde occidental.
Citons pour exemple les travaux de Zhou Huashan sur l'homosexualité en Chine. Chercheur hongkongais et militant pour les droits homosexuels, Zhou cite Mengzi (+/-380-289 av. J.C.), « l'héritier spirituel de Confucius » ${ }^{(4)}$, pour montrer que le sexe n'est pas au centre de la vie des individus : " se nourrir et faire l'amour sont des choses naturelles auxquelles il ne convient pas d'accorder une grande attention ${ }^{(45)}$. Mobilisant cet argument, Zhou explique qu'en Chine, la grande majorité des personnes qui ont des pratiques homosexuelles se marient.

Cette revitalisation de la « culture sexuelle » ancienne (au sens de sexual lore) au service de la modernisation et d'une certaine auto-orientalisation ${ }^{(46)}$, est repérable aussi bien dans des ouvrages universitaires que dans des discours sur la sexualité de locuteurs de culture chinoise ${ }^{(47)}$. Que dire de leur ré-interprétation? Paul E. Festa montre par exemple qu'à Taiwan, le médicament Viagra est aujourd'hui inséré dans la catégorie thérapeutique de médecine traditionnelle des stimulants et des aphrodisiaques. Le médicament biomédical est réapproprié et intégré comme un nouveau remède dans les catégorisations locales pré-existantes à l'innovation.

Les conceptions du corps interviennent dans la construction des représentations de la sexualité. Coexistant avec une conception biomédicale "indigénisée », des conceptions locales, héritées de traditions et d'innovations, sous-tendent les pratiques liées à la sexualité et/ou à la reproduction. Des événements particuliers tels que la

40. Pan Suiming, op. cit., p. 5.

41. Pan, op. cit., p. 13.

42. Robert van Gulik, Sexual Life in Ancient China. A Preliminary Survey of Chinese Sex and Society from ca. 1500 B.C. till 1644 A.D., Leiden, E.J. Brill, 1961.

43. Charlotte Furth, «Rethinking Van Gulik: Sexuality and Reproduction in Traditional Chinese Medicine », in Christina K. Gilmartin, Gail Hershatter, Lisa Rofel and Tyrene White (éds.), Engendering China. Women, Culture and the State, Cambridge (MA), Harvard University Press, 1994, pp. 125-146.

44. Expression empruntée à Anne Cheng. Voir Anne Cheng, Histoire de la pensée chinoise, Paris, Seuil, 1997, p. 159.

45. Zhou Huashan, Beijing tongzhi gushi, Xiangkang, Xiangkang tongzhi yanjiushe, 1996 ; Zhou Huashan, Histoires de " camarades » : les homosexuels en Chine, Paris, ParisMéditerranée, 1997, p. 76.

46. Repérable aussi dans les études portant sur l'indigénisation des études chinoises. Voir aussi le dernier ouvrage de Liu, op.cit., 2005, qui est une comparaison de la « culture sexuelle " (xing wenhua) en Chine et au Japon à travers les arts érotiques. Liu s'intéresse aussi aux objets érotiques (voir note 16).

47. Furth, op.cit:; Charlotte Furth et Ch'en Shu-Yueh, "Chinese Medicine and the Anthropology of Menstruation in Contemporary Taiwan ", Medical Anthropology Quarterly, vol. 6, n 1, 1992, pp. 27-48 ; Farquhar, op.cit.; Paul E. Festa, «The Blue Whirlwind Strikes Below the Belt: Male Sexuality, Gender Politics, and the Viagra Craze in Taiwan », in Catherine S.P. Farris, Lee Anru et Murray A. Rubinstein (éds.), Women in the New Taiwan. Gender Roles and Gender Consciousness in a Changing Society, New York, Sharpe, 2004, pp. 199-220. 
menstruation, la grossesse, la naissance et le mois suivant la naissance sont l'objet de croyances encore très prégnantes au sein des populations de culture chinoise émanant de la médecine traditionnelle ou de la médecine populaire familiale ${ }^{(48)}$. Par exemple, le mois de confinement des femmes après la naissance ou l'éducation prénatale du futur bébé (éducation du foetus: 胎教, taijiao), confirmant la reconnaissance d'une condition foetale de l'être humain ${ }^{(49)}$, sont encore des pratiques très répandues voire systématiques, dans de nombreuses régions et auprès de certaines catégories de populations ${ }^{(50)}$. Ces croyances contribuent à éclairer les constructions locales de la santé sexuelle d'une femme.

Ces événements qui rythment la vie biologique et qui sont l'objet d'un construit social sont conçus comme des périodes de vulnérabilité à la maladie due à une perte conjointe de sang (血, xue) et de force de vie (气, $q i)$. Les diverses recommandations prescrites par la médecine chinoise sous ses formes savantes ou populaires indiquent une préoccupation d'éducation à la prévention de la maladie : elles peuvent apparaitre comme des mesures de protection de la santé des femmes et en particulier de leur santé sexuelle, surtout quand on connaît l'importance attribué au qi dans les représentations du corps, de la vie, de l'environnement et de la vie sociale quotidienne ${ }^{(51)}$. De la même manière, dans les rapports sexuels, l'homme doit éviter d'éjaculer [contenir l'essence vitale jing (精, sperme)] pour se préserver du risque de maladie associé à la déperdition de jing donc de $q i$ [car le jing est du qi transformé]. S'opposant à l'argument de protection de la santé des femmes, d'autres auteurs interprètent ces mêmes prescriptions, qui favorisent le confinement et l'enfermement des femmes, en termes de domination masculine et de rapports sociaux de sexe (genre) dans le cadre d'une conception patrilinéaire et patrilocale de la famille.

\section{Le développement du Sida, l'action de l'Etat et le risque de propagation du VIH par voie sexuelle ${ }^{(52)}$}

Dans la région Asie-Pacifique, 7,4 millions de personnes seraient infectées par le VIH et 1,1 million auraient été nouvellement contaminées en 2003, beaucoup plus que les années précédentes ${ }^{(53)}$. Les faibles taux de séroprévalence dans de nombreux pays de la zone sont trompeurs ; ils masquent des épidémies localisées, en par- ticulier dans les pays les plus peuplés du monde, la Chine et l'Inde.

En Chine, bien que le taux de séroprévalence globale reste faible $(0,07 \%)$ correspondant à 840000 PVVIH (personnes vivant avec le $\mathrm{VIH}$ ) et à 80000 patients malades du Sida en 2003, l'épidémie compte parmi les problèmes de santé publique les plus urgents. Des bouquets de prévalence élevée existent dans certaines régions et parmi des sous-groupes de population. Dans certaines localités du Yunnan, du Xinjiang et du Henan, l'épidémie se généralise : la proportion d'infections par voie sexuelle augmente et le nombre de femmes contaminées s'accrôt rapidement. Entre 2001 et 2002, le nombre de personnes contaminées s'est accru de $44 \%{ }^{(54)}$. Selon le ministère chinois de la Santé, la Chine est entrée dans une phase d'expansion rapide de l'épidémie depuis 1998. La plupart des experts nationaux et internationaux estiment que plus d'un million de personnes seraient infectées. Le ministère de la Santé stipule qu'en 2010, 10 millions de personnes pourraient être séropositives si des moyens de lutte efficaces n'étaient pas mis en ouvre ${ }^{(55)}$.

Depuis 2003, des personnes ont été testées séropositives dans toutes les provinces, régions autonomes et municipalités, montrant une extension géographique de l'épidémie. De plus, l'infection par le VIH se généralise à toute la population. Quatre des six provinces dont les situations épidémiologiques sont les plus préoccupantes sont des régions méridionales (Yunnan, Sichuan, Guangxi, Guangdong), les deux autres étant la province du Xinjiang (nord-ouest) et celle du Henan (région centrale).

Au Guangxi par exemple, le taux d'infection par le VIH parmi les travailleuses du sexe est passé de $0 \%$ en 1996 à

48. Voir, entre autres travaux, ceux de Furth, op. cit.

49. Une reconnaissance peu répandue dans les cultures du monde comme le montre l'étude de Luc Boltansky, La Condition foetale, Paris, Gallimard, 2004.

50. Il est évidemment difficile, voire forcément erroné, de généraliser dans un contexte chinois complexe et diversifié

51. Cf. la revue terminologique des sens du qi, du plus quotidien au plus spécialisé, dans Evelyne Micollier, «Contrôle ou libération des émotions dans le contexte des pratiques de santé qigong », Perspectives chinoises, $\mathrm{n}^{\circ}$ 53, 1999, pp. 22-30.

52. Première partie du texte réactualisé d'Evelyne Micollier, « Phénomènes de stigmatisation dans un monde chinois confronté au VIH-Sida : vers une collaboration entre réponses officielles et société civile », dans L'Approche culturelle de la prévention et du traite ment du VIH-Sida : stigmatisation et discrimination, Paris, UNESCO, Etude spéciale $\mathrm{n}^{\circ} 20,2003$, pp. 39-54.

53. ONUSIDA, Rapport sur l'épidémie mondiale de Sida, 2004.

54. Chen Shengli, Zhang Shikun et Sydney B. Westley, « HIV/AIDS Awareness Is Improving in China ", Asia-Pacific Population Policy, East-West Center, Research Program Population and Health Studies, $n^{\circ}$ 69, 2004.

55. ONUSIDA, HIV-AIDS : China's Titanic Peril 2001, 2002, 89 p. 
$11 \%$ en 2000, indiquant un fort accroissement de la propagation par voie sexuelle ${ }^{(56)} .10 \%$ des personnes recensées porteuses du VIH sont des adolescent(e)s. Une enquête nationale conduite par les autorités sanitaires indique que $60 \%$ des jeunes ne sont pas ou sont mal informés sur la maladie, et que $21 \%$ environ ne connaissent pas du tout les méthodes de prévention.

La majorité des personnes séropositives recensées ont été infectées par voie sanguine - par injection de drogue avec des seringues contaminées, mais aussi par injection et manipulation du sang infecté dans le cadre des structures sanitaires ${ }^{(57)}$. Ceci dit, le nombre d'infections par voie sexuelle connait une augmentation exponentielle : en 1997, 5,5\% et en 2002, 10,9\% du nombre total d'infections par le VIH recensées avaient été contractées par voie hétérosexuelle ${ }^{(58)}$, un taux qui atteint 19,8\% en 2004 et 11,1 \% par voie homosexuelle ${ }^{(59)}$, ce qui porte le taux d'infection par voie sexuelle à $30,9 \%$. Les homosexuels constituent le troisième groupe vulnérable à l'infection par le VIH après les usagers de drogue par voie intraveineuse et les travailleur(se)s du sexe. Cependant, l'ampleur de l'épidémie homosexuelle pourrait être masquée par le fait que $90 \%$ des homosexuels se marient et ont des rapports hétérosexuels avec leur conjoint(e), au moins pour procréer ${ }^{(60)}$.

Dans les années 1990, la propagation de l'épidémie s'est produite dans une première phase par le partage de seringues infectées entre usagers de drogue, puis par voie hétérosexuelle, suivant des modèles épidémiques identiques en Thaillande, au Vietnam, en Birmanie et en Chine du Sud.

Un facteur de vulnérabilité au VIH est la mobilité interne et externe (trans-frontalière) des personnes, ainsi que l'intégration des économies en Asie de l'Est et du Sud-Est qui se traduit par un développement inégal et l'émergence de poches de pauvreté. La mobilité des acteurs de l'industrie du sexe (travailleur(se)s du sexe, clients, propriétaires des divers espaces où se développe l'industrie, proxénètes, passeurs, transporteurs, autorités, etc.) est un facteur de vulnérabilité supplémentaire.

En juin 2002, le rapport de l'ONUSIDA identifiait d'autres facteurs de diffusion de l'épidémie : un système de santé publique en voie de démantèlement - l'exclusion de nombreuses personnes du système de soins et de prévention - et de graves phénomènes de stigmatisation à l'encontre des malades du Sida. A la fin des années 1980, le ministère de la Santé avait pourtant pris des mesures pour réhabiliter le système de santé rural, mais celui-ci s'est dégradé de manière inexorable dans les régions pauvres. Les autorités sanitaires à Pékin ont conscience du problème, et tentent de proposer des solutions visant à rétablir un accès aux soins de qualité pour le plus grand nombre. Malheureusement, ces consignes restent souvent lettre morte localement. En effet, les régions n'ont pas toujours les moyens financiers d'appliquer des mesures au coût élevéé ${ }^{(61)}$.

La pression émanant des organismes internationaux et des Organisation non gouvernementales internationales (ONGI) travaillant en Chine vise à promouvoir une évaluation correcte et une prise de conscience du risque par les différents acteurs concernés (officiels, activistes, organisations locales, acteurs de l'industrie du sexe, famille, adolescents et jeunes adultes, personnel médical).

Par exemple, alors que la vulnérabilité des minorités ethniques est aujourd'hui officiellement reconnue, une reconnaissance attribuable davantage aux pressions des organismes internationaux gouvernements et non gouvernementaux qu'à une réelle volonté politique, les actions de prévention restent peu adaptées à leurs spécificités. Des études pionnières avaient été menées à la suite de la tenue à Pékin en 1995 de la Conférence mondiale sur les femmes ; la mise en œuvre de programmes d'action et de recherche en direction de la santé des femmes des groupes minoritaires avait alors été encouragée ${ }^{(2)}$.

56. Communiqué de presse ONUSIDA : « New UNAIDS Report warns AIDS Epidemic still in early phase and not levelling off in worst affected countries », New York, 2 juillet 2002.

57. Drame de la vente du sang contaminé dénoncé par la presse internationale en 2001 (Elisabeth Rosenthal, «AIDS and Corruption in a Poor Chinese Province », International Herald Tribune, May 31 [New York Times, May 28]): vente de produits sanguins contaminés à des structures sanitaires organisée par des chefs maffieux, un trafic qui a généré une explosion épidémique d'infections par le VIH par voie sanguine dans les régions rurales du centre de la Chine, en particulier dans le Henan ; sur l'organisation de ce commerce, ses implications sociales et économiques, et la responsabilité des autorités locales et nationales, cf. Pierre Haski Le Sang de la Chine. Quand le silence tue, Paris, Grasset, 2005. Bien que le nombre de personnes infectées par le VIH via le commerce du sang et du plasma recensées constitue encore le groupe dominant, ce mode de transmission est peu significatif en termes de prospective épidémique étant donné que la plupart des infections se sont produites avant 1996.

58. ONUSIDA, A Joint Assessment of HIV/AIDS Prevention, Treatment and Care in China, $1^{\text {er }}$ décembre $2003,40 \mathrm{p}$.

59. ONUSIDA, A Joint Assessment of HIV/AIDS Prevention, Treatment and Care in China, $1^{\text {er }}$ décembre $2004,30 \mathrm{p}$

60. Pan Suiming, « Homosexual Behaviors in Contemporary China », Journal of Psychology and Human Sexuality, vol. 7, n 4, 1995, pp. 1-17 ; Pan Suiming, « Male Homosexual Behavior and HIV-related Rrisk in China », in Peter Aggleton (éd.), Bisexuality and AIDS: International Perspectives, Londres, Taylor-Francis Group, Social Aspects of Aids Series, 1996, pp. 178-190 ; Zhou, op. cités, 1996, 2000.

61. Charlotte Cailliez, «L'effondrement du système de santé rural »,Perspectives chinoises, $n^{\circ} 47,1998$, pp. 38-45.

62. Citons par exemple : Fang Tie (éd.), Chuantong wenhua yu xingyu jiankang (Traditional Culture and Reproductive Health), Yunnan shengyu jiankang yanjiu huibian (Yunnan Reproductive Health Research Association), Zhongguo shehui kexue chubanshe (China Social Sciences Press), 1997 ; Tao Chunfang et Xiao Yang (éds.), Zhongguo funü shengyu jiankang yanjiu (Research on Women's Reproductive Health in China), Xin shijie chubanshe (New World Press), 1995. 




Exposition sur le Sida à Pékin. Les lacunes en matière d'éducation sexuelle sont désormais

officiellement reconnues

La ligne d'action dominante consiste à préconiser le maintien des pratiques traditionnelles en matière de santé de la reproduction si elles sont compatibles avec les recommandations globalisées émanant des organismes internationaux, et de rejeter celles qui ont un impact négatif et qui représentent des obstacles à l'application des programmes. Les termes utilisés pour désigner les pratiques et les acteurs évoquent la période maoiste et l'adhésion à un schéma évolutionniste pour caractériser les populations minoritaires, conforme à celui qui était répandu en Union soviétique ; les pratiques de ces populations sont "primitives ", "dépassées ", "pas modernes », etc. Ces aspects de l'intervention gouvernementale mettent en évidence la continuité du discours officiel avec l'ère maoïste inspiré par le schéma de l'évolutionnisme social.

En 2004, des changements notables en matière de politique publique confirment enfin un engagement accru de l'Etat face au risque épidémique du VIH. Les plus notables sont l'élaboration d'un cadre national de soutien (création d'un nouveau comité national Sida par le Conseil d'Etat en février 2004, suivie de la publication du document $\mathrm{n}^{\circ} 7$ en mars 2004 qui définit un cadre global d'intervention), la valorisation de connaissances plus fiables, un intérêt pragmatique pour la compréhension de la dynamique épidémique, et la volonté de développer le traitement et de la prise en charge des personnes vivant avec le VIH. Il s'agit d'avancées considérables par rapport aux périodes précédentes caractérisées par un déni du risque. Alors que la première personne séropositive fut dépistée en 1985, c'est seulement en 1996 que l'ONUSIDA s'implante à Pékin. Dans les années 1990, on observe une prise de conscience progressive par les cadres officiels et/ou les activistes engagés dans la lutte contre le Sida suivie d'actions, mais leurs interventions ne s'appuient pas sur un schème 
national. Aujourd'hui, toutes les organisations engagées dans la lutte et les acteurs sociaux locaux, nationaux ou internationaux travaillant sur le terrain en Chine sont encouragés à coordonner leurs actions en vue d'une plus grande efficacité.

\section{La gestion officielle de la sexualité et de l'intervention de l'Etat dans une perspective diachronique}

Le contrôle de la sexualité par des programmes d'intervention de l'Etat ou la sexualité comme objet de discours officiel à toutes les périodes historiques sont aujourd'hui les objets de débats. Certains auteurs revendiquent une continuité, au-delà des apparences d'émancipation sexuelle des jeunes et de transformations profondes des comportements et du thème de l'économie de marché donnant à voir « une révolution sexuelle » ${ }^{(63)}$. D'autres n'hésitent pas à affirmer que le temps de la régulation de la sexualité par l'Etat est révolu parce que des facteurs tels que la globalisation et la marchandisation sont hors de contrôle des autorités politiques ${ }^{(64)}$; les comportements et les acteurs sont alors considérés comme imprévisibles au-delà des tentatives d'éducation. Ceci dit, comme le soulignent Deborah Davis et Stevan Harrell ${ }^{(65)}$, le retrait de l'Etat de nombreux domaines de la vie quotidienne coïncide avec un contrôle renforcé de la reproduction biologique par la politique de l'enfant unique et des naissances planifiées ${ }^{(66)}$. Revenons néanmoins aux enseignements de l'histoire chinoise. Une lecture diachronique montre que les enjeux sociaux autour de la gestion de la sexualité sont plus importants que la volonté politique d'une gestion efficace de problèmes sanitaires. Des sources attestent de discours et d'actions de l'Etat visant au contrôle des comportements sexuels dès les Han $(-200 /+200)$, première grande dynastie de la Chine impériale ${ }^{(67)}$.

Pendant l'ère républicaine (1912-1949), la sexualité féminine était pensée en termes de reproduction, et on considérait que les femmes n'avaient pas de désir autonome pour le sexe ; par exemple, alors que l'homosexualité masculine était prohibée, l'homosexualité féminine était objet de déni et en conséquence n' avait aucune place dans le discours public ${ }^{{ }^{68)}}$. La normalisation de la sexualité plus que sa répression a été une préoccupation constante pendant les périodes de nationalisme intense comme celle de la première république (révolution de 1911 et chute de l'empire) et la science médicale est apparue comme un guide rationnel de cette régulation ${ }^{(69)}$. Les discours sur la sexualité et les représentations sont marqués par l'évolutionnisme, le nationalisme et le scientisme, attestant une rupture épistémologique par rapport au discours confucéen prévalent jusqu'au début du $X X^{\mathrm{e}}$ siècle ${ }^{(70)}$. Pour les élites intellectuelles de l'époque, le contrôle approprié du désir sexuel est une clé pour restaurer le pouvoir de la nation et réaliser l'objectif de modernisation ${ }^{(71)}$.

Le travail de Harriet Evans sur la Chine maoiste et postmaoiste montre que le sexe et la sexualité sont une cible d'intervention privilégiée des programmes gouvernementaux depuis 1949. Des pratiques telles que la sexualité pré-maritale et extra-maritale, la reproduction, le mariage, la prostitution et l'homosexualité ont été catégorisées ; la monogamie, le célibat, la maternité, l'avortement et la ménopause ont été représentés dans les discours sur l'éducation et la santé sexuelles. Les discours officiels ont alors instrumentalisé la connaissance scientifique pour légitimer l'imposition de prescriptions morales et sexuelles aux femmes : par exemple, dans le climat idéologique contraignant de la Chine d'avant les réformes, les femmes étaient encouragées à devenir des épouses et des mères exemplaires au nom d'une vision essentialiste de la femme considérée avant tout en sa qualité de sexe biologique voué à la reproduction ${ }^{(72)}$.

Selon d'autres auteurs qui contestent le point de vue d'Evans, pendant l'ère post-maoïste, l'idéologie officielle n'a pas activement formulé un discours sur la sexualité des femmes et a donc libéré un espace d'expression et d'action : «le décalage entre l'idéologie officielle et la culture dominante s'est creusé et nombre de sujets [jusque-là passés sous silence] ont été abordés par le discours public... Ceci indique une séparation entre une idéologie officielle plus

63. Harriet Evans, Women and Sexuality in China: Female Sexuality and Gender since 1949, New York, Continuum, 1997.

64. Xu Xiaoqun, op. cit., 1996 ; Farrer, op.cit., 2002.

65. Deborah Davis et Stevan Harrel, Chinese Families in the Post-Mao Era, Berkeley, University of California Press, 1993.

66. Stevan Harrell, "The Anthropology of Reform and the Reform of Anthropology: Anthropological Narratives of Recovery and Progress in China », Annual Reviews of Anthropology, $\mathrm{n}^{\circ}$ 30, 2001, pp. 139-161.

67. Paul R. Goldin, The Culture of Sex in Ancient China, Honolulu, University of Hawaï Press, 2002.

68. Xu, op. cit., p. 406.

69. Frank Dikötter, Sex, Culture and Modernity in China. Medical Science and the Construction of Sexual Identities in the Early Republican Period, Londres, Hurst, 1995, p. 2 et p. 10.

70. Dikötter, op. cit., p. 8

71. Dikötter, op. cit., pp. 1-2.

72. Li Yinhe, Zhongguo nuxing de ganqing yu xing (Love and Sexuality of Chinese Women), Pékin, Jinri zhongguo chubanshe, 1998. 
rigide et une culture dominante plus fluide en matière de régulation et de normalisation de la culture sexuelle postmaoiste » ${ }^{(73)}$. L'Etat a renoncé à imposer des règles sur les comportements sexuels et sur d'autres domaines de la vie privée. Aujourd'hui, le discours officiel exhorte à améliorer la qualité du mariage et à consolider la famille. "Tandis que l'Etat décide encore de ce qui relève de la sphère privée et de la sphère publique, il a laissé un plus large éventail de comportements personnels, tels que les comportements sexuels, dans la sphère privée. Dans le domaine de la sexualité, les femmes et les hommes peuvent adopter des comportements divergents de la norme prescrite durant l'ère maoïste sans subir de pression politique. Le temps d'un ordre moral incontesté en matière sexuelle et autres, imposé par une élite politique et culturelle, est bien révolu » ${ }^{(74)}$.

En fait, dans la Chine des réformes, l'image de la femme et de la sexualité a été transformée pour être en phase avec la nouvelle éthique et le nouveau modèle comportemental du plaisir par la consommation et la marchandisation ; et, face à ce changement, le discours officiel antérieur s'est effacé. Les autorités ont toléré ce changement notamment parce qu'il servait les intérêts économiques nationaux.

Une autre critique d'Evans ${ }^{(75)}$ porte sur le choix de l'auteur de gommer la Révolution culturelle (1966-76). Or, pendant cette période, le discours officiel fait la promotion d'un idéal révolutionnaire du travailleur androgyne, dont l'identité se trouve ailleurs que dans son corps, sa sensualité et sa sexualité. Cet idéal contraste avec les autres périodes de l'histoire de la République populaire au cours desquelles la différence entre l'homme et la femme est essentialisée.

Aujourd'hui, les jeunes âgés de 10 à 24 ans représentent $26 \%$ de la population totale, soit 327 millions de personnes (recensement de l'an 2000), d'où l'importance des études sur la sexualité des jeunes face au risque d'IST et de Sida. De plus, la prévalence des rapports pré-maritaux oscille entre $44 \%$ et $91 \%$ selon des statistiques récentes de l'Association nationale de la planification familiale ${ }^{(76)}$. Son directeur souligne que les activités sexuelles des personnes non mariées et leurs connaissances en matière de sexualité ne sont pas prises en compte dans les enquêtes. Les programmes de santé sexuelle ne sont toujours pas dirigés vers les jeunes non mariés, ce qui représente une grave lacune en matière de prévention et d'éducation sexuelle.

La lecture des manuels d'éducation sexuelle ${ }^{(77)}$ révèle que leurs auteurs considèrent la masturbation et les rapports prémaritaux comme des questions secondaires alors qu'en revanche, la moralité sexuelle occupe une place-clé dans les contenus ${ }^{(78)}$. Aujourd'hui, les cours d'éducation sexuelle sont encore optionnels dans les institutions scolaires, intégrés au programme de biologie et peu orientés vers l'apprentissage des « techniques de planification de la vie » (Health Life Planning Skills). Il faut signaler l'initiative expérimentale de l'Association nationale de la planification familiale qui a produit des manuels pour l'éducation des adolescents en collaboration avec le Fonds des Nations unies pour la population (FNUAP) ${ }^{(79)}$. Le programme prévoit la diffusion de ces ouvrages dans tout le pays et la formation des adolescents au niveau local dans la mesure du possible, c'est-àdire si la résistance des acteurs sociaux (parents, professeurs et représentants du gouvernement) ne fait pas obstacle à sa mise en œuvre.

En reformulant ses programmes d'éducation sexuelle, l'Etat pourrait engager des actions interactives dans le cadre familial. En effet, espace privilégié d'apprentissage pour ses jeunes membres, la famille est également un lieu d'éducation pour les membres des autres générations. Relais essentiel des consignes officielles à côté de l'école et des lieux de travail, le contexte familial est le cadre normatif traditionnel et accepté par tous de communication avec les femmes. Il pourrait se révéler un cadre approprié pour des interventions

73. Xu, op. cit., p. 406.

74. Xu, op. cit., p. 407.

75. Elaine Jeffreys, book review of Women and Sexuality in China: Female Sexuality and Gender since 1949, New York, Continuum, 1997, by Harriet Evans, China Journal, vol. 40, 1998, pp. 207-210.

76. Xinhua (agence de presse Chine nouvelle), « Research shows Premarital Sex Prevalent in China » (Statistics released by The Chinese Family Planning Association), 10 octobre 2003.

77. Yao Peikuan, «The Study and Practice of Adolescent Sex Education in China », Shanghai, Shanghai kexue yuan, Shanghai Academy of Social Sciences Papers, vol. 4, 1992, pp. 443-56 ; Yao Peikuan (éd.), Survey on Sex Education: The Report, Xuelin, Xuelin Publishing House, 1990 ; Yao Peikuan (éd.), Education for Adolescence, Guangxi Science and Technology Publishing House, 1989 ; Yao Peikuan (éd.), A Common Sense Reader for Adolescents, Shanghai, Shanghai People's Publishing House, 1988; Zhu Weibing (éd.), One Hundred Questions on Adolescence for Middle School Students, Shanghai, Shanghai Medical College Publishing House, 1988 ; She Jianhai, «Woguo dangdai qingchunqi xing jiaoyu yanjiu he shixian » (Recherche et pratique de l'éducation sexuelle pour les jeunes en Chine contemporaine), in Shanghai jiaoyu keyan (Recherche scientifique sur l'éducation sexuelle de Shanghai), n 1, 1988 ; Wu Jieping (éd.), Xing Yixue (Sexologie médicale), Wenxian, Kexue jishu chubanshe, 1988 ; Sports Hygiene Department of the National Education Commission, One Hundred Questions and Answers on Sex Education for Middle School Students, Pékin, Beijing University Publishing House, 1988.

78. Liu Dalin, «The Development of Sex Education in China », in Linda Jaivin (éd.), « Sex », Chinese Sociology and Anthropology, vol. 27, $n^{\circ}$ 2, 1994-1995, pp. 10-36; Voir les tableaux comparatifs des contenus de divers manuels dans une perspective diachronique, Liu et al., 1997, p. 555 (période d'introduction : 1952-1978), p. 561 (période de promotion : 1988-années 1990).

79. Cf. Zhongguo jihua shengyu xiehui (China Family Planning Association)/United Nations Population Fund (UNFPA), Qingshaonian rensheng jineng peixun zhinan (Guidebook for Adolescent Reproductive Health Life Planning Skills Training), Pékin, 2003, et un manuel destiné aux formateurs, Qingshaonian shengzhi jiankang gongzuozhi peixun zhinan (Guidebook for Training of ARH Trainers), Pékin, 2003. 
visant à l'éducation pour la santé, ainsi qu'à la prévention et à la prise en charge du Sida et des IST.

En Chine, le développement de la recherche sociologique et ethnologique sur la sexualité s'est accéléré depuis 1990. Cette évolution révèle en même temps qu'elle permet des changements en matière de pratiques et de représentations et l'accroissement de la tolérance par rapport à la politique maoïste puritaine en matière de morale sexuelle. Le risque de transmission sexuelle du VIH et l'augmentation du nombre de personnes infectées par des IST en général a favorisé le développement de ces études. En effet, certains représentants de l'Etat, bien que critiqués par d'autres, ont souhaité disposer d'instruments de connaissance afin de pouvoir définir des priorités et engager une action. La mobilisation collective contre le Sida initiée par des acteurs internationaux, non gouvernementaux ou non directement gouvernementaux comme les $\mathrm{GONGOs}^{(80)}$, a également contribué au développement de ces recherches ${ }^{(81)}$. Toutefois, la gestion de la sexualité et du risque sanitaire par l'Etat s'inscrit toujours dans un schéma évolutionniste de modernisation et de progrès, en continuité avec l'idéologie moderniste et essentialiste de la Chine républicaine. Les actions et les discours officiels confirment cette tendance en dépit de la pluralisation et de la globalisation de la société. Comme le montre le débat entre spécialistes chinois et non chinois de la sexualité en tant que pratique sociale, des forces contradictoires, identifiées ici à partir des changements repérables dans les pratiques et les représentations de la sexualité sont en présence. Les tensions et les négociations qui s'opèrent entre les différentes catégories d'acteurs et les contradictions symboliques, générées par exemple par l'éclectisme des représentations de la jeunesse urbaine, ne sont pas stabilisées. Il est donc trop tôt pour appréhender sur le long terme la pertinence du projet étatique et son impact sur les comportements individuels. •

80. «Government-Organised Non-Government Organisation » est un terme proposé par Gordon White, Jude Howell et Shang Xiaoyuan (éds.), In Search of Civil Society: Market Reform and Social Change in Contemporary China, Oxford, Clarendon, 1996. Il s'agit d'une forme d'organisation sociale spécifique au contexte chinois qui joue le rôle d'institution médiatrice entre les institutions nationales et internationales ; sur les GONGOs engagées dans la lutte contre le Sida, cf. Micollier, op. cit., 2003, pp. 49-51.

81. Evelyne Micollier, «Acteurs de la mobilisation collective contre le Sida en Chine et solidarités transnationales : dynamiques locales et visibilité des groupes de défense des minorités sexuelles et sociales », dans Fred Eboko, Frédéric Bourdier, Pierre Broqua et Olivier Fillieule (éds.), "Mobilisations collectives face au Sida dans le monde. Translations internationales et dynamiques locales » Face à face. Regards sur la santé, $\mathrm{n}^{\circ} 7,2005$, pp. 31-40, revue en ligne www.ssd.u-bordeaux $2 . \mathrm{fr} / \mathrm{faf}$ 